\title{
Retroperitoneal neurogenic tumor diagnosed by endoscopic ultrasonography
}

\author{
Nuno Veloso ${ }^{1}$, Pedro Figueiredo ${ }^{1}$, Pedro Pinto-Marques ${ }^{1}$, Ana Reis ${ }^{1}$, María José Brito ${ }^{2}$ and João Freitas ${ }^{1}$ \\ Departments of ${ }^{1}$ Gastroenterology and ${ }^{2}$ Pathology. Hospital Garcia de Orta. Almada, Portugal
}

\section{INTRODUCTION}

The retroperitoneum can host a wide spectrum of pathologies, ranging from rare benign tumors to malignant neoplasms (primary or metastatic). Malignant tumors are four times more frequent than benign, being neurogenic tumors one of the most common benign pathologies (1).

\section{CASE REPORT}

A 57-year-old woman presented to our department with a several-months history of right upper quadrant pain. Physical examination and laboratory evaluation was unremarkable. On abdominal ultrasound, a retroperitoneal solid mass adjacent to the posterior surface of the pancreatic neck was shown. To further characterize the lesion, an abdominal magnetic resonance imaging was performed which revealed a well-demarcated round $35 \mathrm{~mm}$ mass, located in retroperitoneum, below the emergence of the celiac trunk, shaping the posterior surface of the pancreatic neck (Fig. 1).

The patient was referred for an endoscopic ultrasonographic fine needle aspiration (EUS-FNA). A well delineated, Doppler negative, retroperitoneal $30 \mathrm{~mm}$ solid mass was seen, adjacent to posterior surface of the pancreatic neck at the level of

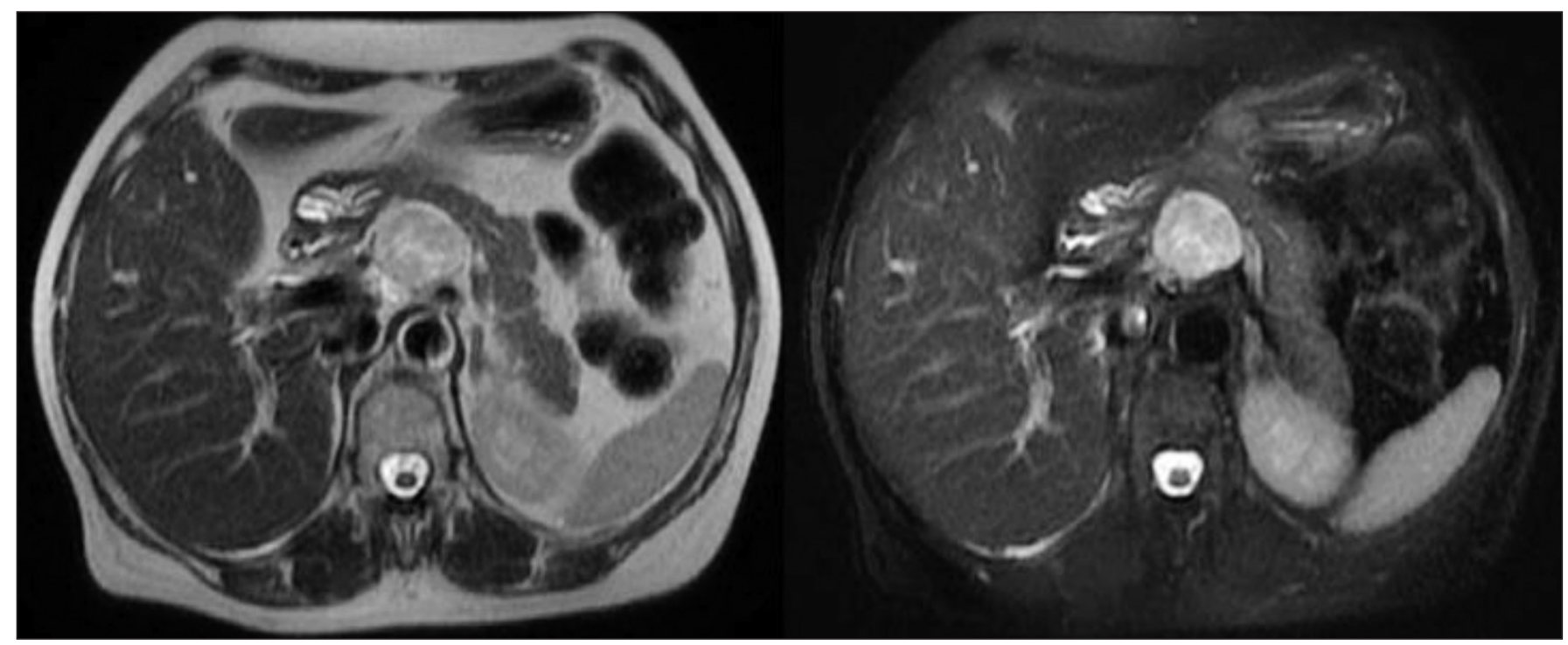

Fig. 1. MRI demonstrated a well-demarcated round $35 \mathrm{~mm}$ mass in retroperitoneum, hypointense signal on T1-weighted images and slightly heterogeneous hyperintense on T2-weighted images. 


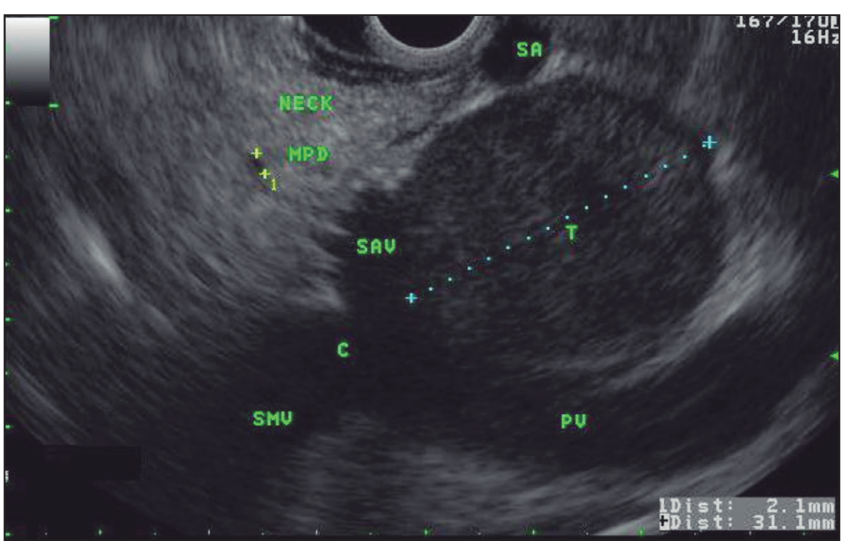

Fig. 2. EUS (C: portosplenic confluence; MPD: main pancreatic duct; PV: portal vein; SA: splenic artery: SAV: splenic vein; SMV: superior mesenteric vein; T: tumor).

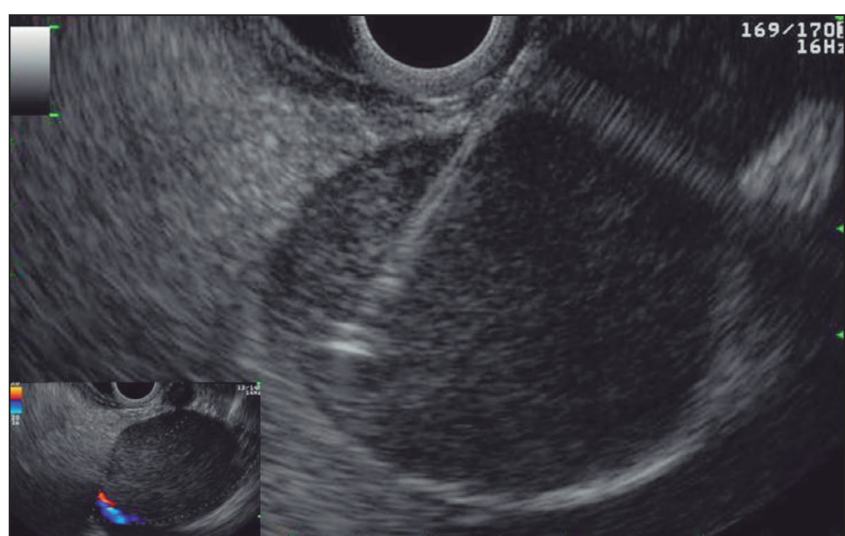

Fig. 3. Fine needle aspiration.

the portosplenic confluence (Fig. 2). A EUS-FNA was performed (Olympus EZ-ShotTM; 22G; 4 passes) (Fig. 3). Cytology showed clusters of eosinophilic cells, spindle-shaped, uniform nuclei, without mitoses. Immunohistochemistry performed on the cell block was positive for S-100 and vimentin, compatible with neural tissue (Fig. 4).

\section{DISCUSSION}

Tumors of the retroperitoneum are rare, and neurogenic tumors comprise only 1-10\% (2). Benign neurogenic tumors are discovered as an incidental finding during imaging for unrelated symptoms (3). There are no specific tumor markers or characteristic imagiology. EUS-FNA could be an effective and minimal invasive choice for the definitive diagnosis and tissue sample. Radiological surveillance in asymptomatic patients or surgical resections in symptomatic patients are the options for treatment.

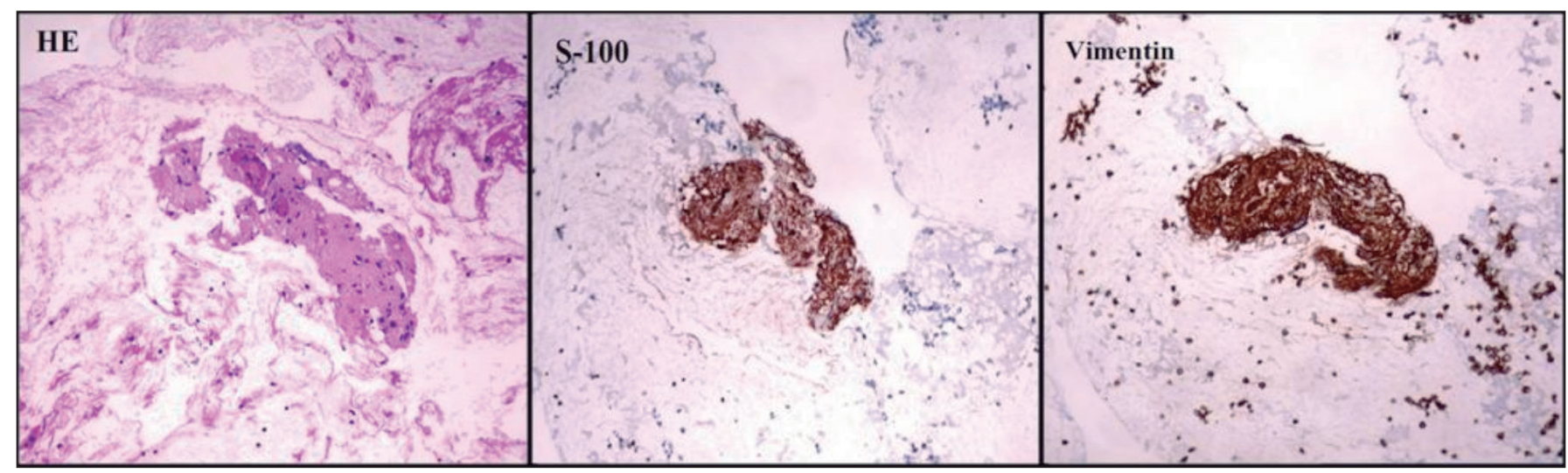

Fig. 4. Cytology and immunohistochemistry.

\section{REFERENCES}

1. Van Roggen JF, Hogendoorn PC. Soft tissue tumours of the retroperitoneum. Sarcoma 2000;4:17-26.

2. Lane RH, Stephens DH, Reiman HM. Primary retroperitoneal neoplasms: CT findings in 90 cases with clinical and pathologic correlation. AJR Am J Roentgenol 1989;152:83-9.

3. Strauss DC, Hayes AJ, Thomas JM. Retroperitoneal tumours: Review of management. Ann R Coll Surg Engl 2011;93:275-80. 